\title{
On the Three-Dimensional Transformations founded on the Twisted Cubic and its Chord System.
}

\author{
By Dr J. F. Tinto. \\ (Read and Received 10th March 1916).
}

\section{SeCtion I.}

The base transformations which transform the chord system of one twisted cubic into the chord system of another.

$\S 1$. We shall suppose that there are two distinct spaces denoted by (1) and (2) containing twisted cubics $\Sigma_{3}$ and $\Sigma_{3}^{\prime}$ respectively.

An endless series of bi-rational point transformations exists, each of which has the property of transforming a chord of $\Sigma_{3}$ into a unique chord of $\Sigma_{3}{ }^{\prime}$, and vice versa.

In all, except the first, a finite number of chords exists (the number depending on the order of the transformation) which have no unique correspondents, but an infinite number constituting a rational ruled surface. Such chords will be referred to as fundamental (F-) chords.

The order of the general transformation of the series is $2 p-1$, where $p$ is any positive integer. The case $p=1$ is a collineation in which $\Sigma_{3}$ and $\Sigma_{3}^{\prime}$ are corresponding curves. $p=2$ gives a cubic transformation having $3 F$-chords of first order, $\Sigma_{3}, \Sigma_{3}^{\prime}$ being F-curves of order 1 . We shall say that a chord (or the cubic itself) is of order $r$ when its corresponding surface is of order $2 r$. This is the case of paramount importance, since all the others can be derived from it. $p=3$ gives a quintic transformation having $5 F$-chords in each space, one being of order 2 and four of order 1, whilst $\Sigma_{3}, \Sigma_{3}^{\prime}$ are $F$-curves of order 2 . This transformation is the product of two cubic transformations of type $p=2$.

Generally the transformation of order $2 p-1$ can be derived by applying in succession transformations of type $p=2$. It will be denoted by $T_{s p-1}$. 
$\S 2$. Consider a cubic transformation having $3 F$-chords,

$$
{ }_{1} L_{1},{ }_{1} L_{2},{ }_{1} L_{3} \text { in (1) and }{ }_{1} L_{1}^{\prime},{ }_{1} L_{2}^{\prime},{ }_{1} L_{3}^{\prime} \text { in (2). }
$$

It can be built up by means of pencils of planes through ${ }_{1} L_{1},{ }_{1} L_{2},{ }_{1} L_{3}$ projectively related to pencils of planes through ${ }_{1} L_{1}{ }^{\prime},{ }_{1} L_{2}{ }^{\prime},{ }_{1} L_{3}^{\prime}$ respectively.

Corresponding to a point on ${ }_{1} L_{1}$ we get a line meeting ${ }_{1} L_{2}^{\prime},{ }_{1} L_{3}^{\prime}, \Sigma_{3}^{\prime}$ and corresponding to ${ }_{1} L_{1}$ as a whole, a conicoid determined uniquely by ${ }_{1} L_{2}{ }_{1}, L_{3}^{\prime}, \Sigma_{3}^{\prime}$.

This conicoid may be regarded as generated by chords of $\Sigma_{3}^{\prime}$ which intersect any one of the lines meeting ${ }_{1} L_{2}{ }_{2},{ }_{2} L_{3}, \Sigma_{3}{ }^{\prime}$. We may therefore look upon, $L_{1}$ as having for its correspondent an infinite number of chords which constitute a ruled surface of the second order.

Corresponding to a point on $\Sigma_{3}$ we get a line intersecting ${ }_{1} L_{1}^{\prime},{ }_{1} L_{2}^{\prime},{ }_{1} L_{3}^{\prime}$ and corresponding to $\nu_{3}$ as a whole, the conicoid uniquely determined by ${ }_{1} L_{1},{ }_{1} L_{2},{ }_{1} L_{3}^{\prime}$.

A line of space (1) in general transforms into a twisted cubic in (2), which intersects $L_{1} L_{1},{ }_{1} L_{2}^{\prime},{ }_{1} L_{3}^{\prime}, \Sigma_{3}^{\prime}$ each in 2 points. It follows that any chord of $\Sigma_{3}$ (with the exception of the $F$-chords) transforms into a unique chord of $\Sigma_{3}^{\prime}$, and conversely.

Let a point $P$ in (1) describe a line; the chords through $P$ generate a rational ruled quartic surface having $\Sigma_{3}$ as double curve. This quartic transforms into a rational ruled octic in (2), having $\Sigma_{3}^{\prime}$ as curve of multiplicity 4 , and ${ }_{1} L_{1}{ }^{\prime},{ }_{1} L_{2}{ }^{\prime},{ }_{1} L_{3}{ }^{\prime}$ as double lines.

We therefore have what may be called a quadratic transformation between the chord systems of $\Sigma_{3}, \Sigma_{3}^{\prime}$ regarded as two dimensional systems of lines. It can be built up directly by means of pencils of conicoids through $\left({ }_{1} L_{1}, \Sigma_{3}\right),\left({ }_{1} L_{2}, \Sigma_{3}\right),\left({ }_{1} L_{3}, \Sigma_{3}\right)$ projectively related to pencils through $\left({ }_{1} L_{1}^{\prime}, \Sigma_{j}^{\prime}\right),\left({ }_{1} L_{i 2}^{\prime}, \Psi_{3}^{\prime}\right)$, etc., respectively. It is of interest to note that in this two-dimensional geometry the primary element is a chord, and the secondary a conicoid through the cubic. Any projected theorem in ordinary plane geometry can be transferred to this system by changing the names point, line, curve of order $r$, into chord, conicoid, ruled surface of order $2 r$ respectively. The principle of duality also applies; indeed, so far as projective properties are concerned, the two geometries may be regarded as identical. 
$\$ 3$. We can now form the product of successive cubic transformations. To do this we imagine that there are $q$ spaces, each containing one $F$-twisted cubic and no other $F$-curve.

Applying a cubic transformation between (1) and (2), and then between (2) and (3), and so on, we can obtain by proper choice of the $F$-chords a trunsformation of order $2 p-1$ between the first and last spaces, which possesses the property of transforming a chord of $\Sigma_{3}$ into a chord of $\Sigma_{3}^{\prime}$.

We shall suppose that there are $\alpha_{i} H^{\prime}$-chords of order $i$ where $i$, as will be shown presently, may have any value from 1 to $p-1$. These chords will be denoted by ${ }_{i} L_{r}\left(r=1,2, \ldots \alpha_{i}\right)$.

Corresponding to a point on ${ }_{i} L_{r}$ we get a curve of order $i$ rational since it is obtained by successive transformations of a line. It must lie on a ruled surface of order $2 i$, rational also, since it is obtained by successive transformations of a conicoid. This ruled surface, which can be regarded as generated by chords of $\mathrm{S}_{3}^{\prime}$, is the correspondent of ${ }_{i} L_{r}$. It has $\mathcal{Z}_{3}^{\prime}$ as a curve of multiplicity $i$, and will be denoted by ${ }_{r} S_{i}$.

Corresponding to a point on $\breve{s}_{3}$ we get a rational curve of order $p-1$ meeting $\Sigma_{3}^{\prime}$ in $p-2$ points, and corresponding to $\searrow_{3}$ as a whole, a rational surface of order $2(p-1)$ which has $\Psi_{3}^{\prime}$ as curve of multiplicity $p-2$. Since the cross-ratio of four points on a chord of $\dot{\Sigma}_{3}$ is unaltered by the cubic transformation, the same result will be true of $T_{2 p-1}$.

$\$ 4$. A rational ruled quartic in (1) having $\searrow_{3}$ as double curve is transformed by $T_{3,-1}^{Y}$ into a rational ruled surface of order $4 p$, having $\nu_{i}^{\prime}$ as curve of multiplicity $2 p$, and ${ }_{i} L_{r}^{\prime}$ as líne of multiplicity $2 i$. These surfaces will be denoted by $S_{4}, S_{4 p}^{\prime}$ '.

Any plane section of $S_{4 p}^{\prime}$ is a curve of order $4 p$ having 3 nodes of order $2 p$ and $\alpha_{i}$ nodes of order $2 i$. It must be unicursal. For choose two arbitrary planes, $\pi$ and $\pi^{\prime}$, in (1) and (2) respectively, $\pi$ cuts $S_{4}$ in a unicursal plane quartic, and since to each generator of $S_{4}$ there corresponds in general a unique generator of $S_{4 p}$, we can establish a $(1-1)$ point correspondence between the two plane curves. The section of $S_{4 p}{ }^{\prime}$ must, therefore, be unicursal. $T_{2 p-1}$ in fact establishes between the points of the planes $\pi$ and $\pi^{\prime}$ a Cremona transformation of order $4 p$. 
For corresponding to a line in $\pi$ we get a unicursal curve of order $4 p$ in $\pi^{\prime}$. The $F$-points of this plane transformation are of course the meets of the $F$-chords and of the cubics $\Sigma_{3}, \Sigma_{3}^{\prime \prime}$ with $\pi$ and $\pi^{\prime}$. If $\Sigma_{3}$ and $\Sigma_{3}^{\prime}$ meet $\pi$ and $\pi^{\prime}$ in $A B C, A^{\prime} B^{\prime} C^{\prime}$ respectively, the lines $A B, B C, C A, A^{\prime} B^{\prime}, B^{\prime} C^{\prime}, C^{\prime} A^{\prime}$ are $F$-lines of the first order, and the corresponding points $F$-points of the same order. If the chord ${ }_{i} L_{r}$ meet $\pi$ in ${ }_{i} D_{r}$, the correspondent of ${ }_{i} D_{r}$ is a unicursal curve of order $2 i$, which is a plane section of,$S_{i}$, the surface which corresponds to ${ }_{i} L_{r}$. The $F$-surfaces corresponding to the $F$-chords are, therefore, such that any plane section is a unicursal curve.

$T_{2 p-1}$ consequently establishes a transformation of order $p$ between the chord systems of $\Sigma_{3}, \Sigma_{3}^{\prime}$. It follows that the transformation of order $p$ is the product of successive quadratic transformations. We may, therefore, expect that the number and order of the $F$-chords is the same as the number and order of the $F$-points of a Cremona transformation of order $p$.

Forming the Cremona equations for the transformation of order $4 p$ between $\pi$ and $\pi^{\prime}$ we get

$$
\sum \alpha_{i}(2 i)^{2}+3(2 p)^{2}+3=(4 p)^{2}-1
$$

and

$$
\Sigma \alpha_{i} 2 i+3(2 p)+3=3(4 p-1),
$$

giving

$$
\Sigma \alpha_{i} i^{2}=p^{2}-1 \text { and } \Sigma \alpha_{i} i=3(p-1),
$$

which are the Cremona equations for a transformation of order $p$. Our conclusion is therefore verified.

The result may be stated thus: Corresponding to any Cremona transformation of order $p$ between two plane systems, there exists a space transformation of order $2 p-1$ having twisted cubics $\Sigma_{3}, \Sigma_{3}^{\prime}$ as $F$-curves of order $p-1$ and as $F$-lines, chords of these cubics whose number and order is the same as the number and order of the $F$-points.

Since any Cremona transformation can be resolved into the product of quadratic transformations, it follows that any space transformation whose $F$-curve system is composed only of a twisted cubic and chords of that cubic, can be resolved in to the product of cubic transformations of type $p=2$. The plane Cremona transformations are in fact identical with the transformations of the chord system of a twisted cubic. This we may regard as a ccnsequence of the remark at the end of $\S 2$. 
\$5. Two particular cases are of interest:-

(a) Suppose that the twisted cubics $\Sigma_{3}, \Sigma_{3}^{\prime}$ are degenerate and composed of a line $\sigma_{1}, \sigma_{1}^{\prime}$ and a conic $\Sigma_{2}, \Sigma_{2}^{\prime}$, the line meeting the conic in 1 point.

If $P$ describe a line in (1), the chords through it generate a rational ruled cubic $S_{s}$, having $\sigma_{1}$ as double line and $\Sigma_{2}$ as simple curve. This transforms into a rational surface of order $4 p-1$, $S_{4 p-1}^{\prime}$ having $\Sigma_{2}^{\prime}$ as curve of multiplicity $2 p-1, \sigma_{1}^{\prime}$ as line of multiplicity $2 p$, and ${ }_{i} L_{r}$ as line of multiplicity $2 i$. The Cremona transformation between planes $\pi$ and $\pi^{\prime}$ is now of order $4 p-1$. There is one $F$-point of order $2 p$, two of order $2 p-1, a_{i}$ of order $2 i$ $(i=1,2, \ldots p-1)$, and two of order 1 . The number and order of the $F$-chords are the same as before.

(b) Suppose that the conic $\Sigma_{2}$ is also degenerate and composed of two lines $\sigma_{2}, \sigma_{3}$, only one of which (say $\sigma_{2}$ ) intersects $\sigma_{1}$. When $P$ describes a line in (1), the chords through it (meeting $\left.\sigma_{1}, \sigma_{3}\right)$ generate a conicoid through $\sigma_{1}, \sigma_{i ;}$. This transforms into a rational ruled $S_{s p-2}$, having $\sigma_{1}^{\prime}, \sigma_{3}^{\prime}$ as lines of multiplicity $2 p-1$ and $\sigma_{2}^{\prime}$ as line of multiplicity $2 p-2$. The Cremona transformation is of order $4 p-2$, and has two $F^{\prime}$-points of order $2 p-1$, one of order $2 p-2, a_{i}$ of order $2 i(i=1,2, \ldots p-1)$, and one of order 1 . The number and order of the $F$-chords are as before.

\section{Superposed Spaces.}

\section{§6. Involutive Base Transformations.}

The spaces (1) and (2) may be considered not as separate but as one space. Suppose $\Sigma_{3}, \Sigma_{3}{ }^{\prime}$ to coincide, and let ${ }_{i} L_{r}$ coincide with " $L_{r}^{\prime}$ for all values of $i$ and $r$. The transformation $T_{2 p-1}$ can now be completely involutive. The transformation of the chord system into itself will, in this case, be also completely involutive. Such a transformation can possess a surface of self-corresponding chords. The following involutive transformation is analogous to a de Jonquières transformation, which is perspective in character :-

Consider a ruled surface $M$ of order $2 p$ having a chord $L$ of $\Sigma_{3}$ as line of multiplicity $p-2$ and $\check{\zeta}_{3}$ as a curve of multiplicity $p$. Any conicoid of the pencil determined by $L \Sigma_{3}$ meets this surface $M$ in two chords, $R$ and $S$. 
If $\lambda$ be a chord, the conicoid uniquely determined by $L \lambda \Sigma_{2}$ meets $M$ in chords $R$ and $S$, say, the harmonic conjugate $\lambda$ of $\lambda$ with respect to $R$ and $S$ on this conicoid is taken to be the involutive correspondent of $\lambda$.

The order of the transformation is $p$, and the surface $M$ is a surface of self-corresponding chords.

Corresponding to a rational $S_{4}$ we get a rational ruled $S_{4 p}{ }^{\prime}$ having $L$ as line of multiplicity $2(p-1)$ and possessing $2(p-1)$ fixed chords as double lines. The locus of the harmonic conjugates of $L$ with respect to $M$ is a surface of order $2(p-1)$, which meets $M$ in the $2(p-1)$ fixed chords. This surface corresponds to $L$. $L$ is a $F$-chord of order $p-1$. Corresponding to each of the $2(p-1)$ chords we get the conicoid through it and $L \Sigma_{3}$. Such chords are the remaining $F$-chords of order 1 .

\section{SeCtion II.}

$\$ 7$ The base transformations will now be used to build up more general transformations which have the property of transforming the chord system of $\Sigma_{3}$ into the chord system of $\Sigma_{3}$.

We shall suppose the base transformation to be of order $2 p-1$ and to have $\alpha_{i} F$-chords of order $i$ where

$$
\sum_{i=1}^{i=p-1} \alpha_{i} i^{2}=p^{2}-1 \text { and } \sum_{i=1}^{i=p-1} \alpha_{i} i=3(p-1) \text {. }
$$

If a $F$-curve of order $i$ intersect a $F$-chord of order $j$ in $\beta_{i j}$ points, then

$$
\sum_{j=1} i \beta_{i j}=p i \text { and } \sum_{j=1} \beta_{i j}=3 i-1 .
$$

The following theorem will be required frequently and will be quoted as A.

If a rational surface of order $2 m$ generated by chords of a twisted cubic, which is a curve of multiplicity $m$ on it, have a rational space curve of order $s$ on it meeting each generacor once, this curve must intersect the cubic in $2 s-m$ points.

Further, two rational curves of orders $r$ and $s$ intersect in $r+s-2 m$ points.

Consider a surface of order $(-p+2) n-(2 p+4)$ in (1) having $\Sigma_{3}$ as a curve of multiplicity $(p+1) n-(p+3)$ and a $F$-chord ${ }_{i} L_{r}$ as a line of multiplicity $i(n-1)$, where $n$ is any integer. 
Transforming into (2) we get by $T_{2 p-1}$ a surface of order

$$
\begin{gathered}
(2 p-1)[(2 p+2) n-(2 p+4)]-2(p-1)[(p+1) n-(p+3)] \\
-\sum_{i=1} 2 \alpha_{i} i^{2}(n-1)
\end{gathered}
$$

giving on reduction $(2 p+2) n-(2 p+4)$.

The transformed surface is of the same order and precisely the same nature as the original surface. They will be denoted by ${ }_{n} U_{p},{ }_{n} U_{p}^{\prime}$ respectively.

${ }_{n} U_{p}^{\prime}$ contains $\Sigma_{3}^{\prime}$ as a curve of multiplicity $(p+1) n-(p+3)$ and the $F$-chords ${ }_{i} L_{r}^{\prime}$ as lines of multiplicity $i(n-1)$.

The curve of order $i$ corresponding to a point on ${ }_{i} L_{r}$ ' meets ${ }_{n} U_{p}$ in $i[(2 p+2) n-(2 p+4)]$ points. Of these $i[(p+1) n-(p+3)]$ lie on $\Sigma_{3}$, and

But

$$
\sum_{j=1} \beta_{i j} i(n-1) \text { on the } F \text {-chords. }
$$

$$
\sum_{j=1} \beta_{i j} i(n-1)=p i(n-1),
$$

hence the number of proper points of intersection is $i(n-1)$, and hence ${ }_{i} L_{r}^{\prime}$ is a line of multiplicity $i(n-1)$ on ${ }_{n} U_{p}^{\prime}$.

Let an arbitrary point $P$ be taken in (1) and let $e$ be the chord of $\Sigma_{3}$ through it. Let $P_{1}^{\prime}$ and $e^{\prime}$ be the corresponding point, and chord in (2). $e$ meets ${ }_{n} U_{p}$ in two points $R$ and $S$, and $e^{\prime}$ meets ${ }_{n} U_{p}^{\prime}$ in the two corresponding points $R^{\prime}$ and $S^{\prime}$. Let $P_{0}, P^{\prime}$ be chosen on $e, e^{\prime}$ so that $\left(P, P_{0}, R, S\right)=\left(P_{1}^{\prime}, P^{\prime}, R^{\prime}, S^{\prime}\right)=-1$. Since the cross-ratio of 4 points on a chord is unaltered, $P^{\prime}$ corresponds to $P_{0}$ in $T_{2 p-1}$.

In the new transformation we shall take $P^{\prime}$ as the correspondent of $P$. The correspondence is $(1-1)$, and reversible.

The transformation to which it gives rise is reversible and of order $(2 p+2) n-3$.

Let $P$ describe an arbitrary line in (1). $P_{0}$ describes the harmonic locus of this line with respect to ${ }_{n} U_{p}$, while $P_{j}^{\prime}$ describes the correspondent of the line by $T_{2 p-1}$, namely, a curve of order $2 p-1$. $P^{\prime}$ traces out the harmonic locus of this curve with respect to ${ }_{n} U_{p}$. These harmonic loci are corresponding curves in $T_{2 p-1}$.

The harmonic locus described by $P_{0}$ is a curve of order

$$
(2 p+2) n-(2 p+4)+3 \text {, i.e. }(2 p+2) n-(2 p+1),
$$

and since it lies on a $S_{4}$ it must meet $\Sigma_{3}$ in

$$
2[(2 p+2) n-(2 p+1)]-2 \text { points by } A \text {. }
$$


Transforming by $\eta_{z p-1}$ we get a curve of order

$$
\begin{gathered}
(2 p-1)[(2 p+2) n-(2 p+1)]-2(p-1)[(2 p+2) n-(2 p+1)] \\
+2(p-1),
\end{gathered}
$$

giving on reduction

$$
(2 p+2) n-3 .
$$

This curve lies on a $S_{4 p}{ }^{\prime}$, and it must by $\mathrm{A}$ meet $\Sigma_{3}{ }^{\prime}$ in

$$
2[(2 p+2) n-3]-2 p \text { points, }
$$

or

$$
(4 p+4) n-(2 p+6) \text { points. }
$$

It must also intersect the chords ${ }_{i} L_{r}^{\prime}$ in $2 i$ points each. It is rational since the generators of $S_{4 p}{ }^{\prime}$ establish a $(1-1)$ correspondence between its points and those of a plane section of $S_{4 p}$.

Corresponding to a line in (2) we get, in the same way, a rational curve of order $(2 p+2) n-3$ meeting $\Sigma_{3}$ in

$$
(4 p+4) n-(2 p+6) \text { points an }{ }_{i} L_{r} \text { in } 2 i \text { points. }
$$

\section{\$ 8. The F-systems.}

If a chord of $\Sigma_{3}$ touch ${ }_{n} U_{\mu}$, its corresponding chord will touch ${ }_{n} U_{p}^{\prime}$. Corresponding to any point on the first chord we get the point of contact of the second, and vice versa. These chords are therefore $F$-lines of the transformation, and their points of contact $F$-points. The chords of $\Sigma_{j}$ which touch ${ }_{n} U_{p}$ generate a ruled $F$-surface and their points of contact a $F$-curve.

To find the order of the surface, we have to find the number of tangent-chords which can beidrawn from a point on $\aleph_{j}$ to ${ }_{n} U_{p}$. This number is $(2 p+2) n-(2 p+2)$, and consequently the order of the surface is $(4 p+4) n-(4 p+4)$. It has $\Sigma_{3}$ as a curve of multiplicity $\left({ }^{2} p+2\right)(n-1)$, and ${ }_{i} L_{r}$ as line of multiplicity $2 i(n-1)$. It will be denoted by ${ }_{n} W_{n}$. The curve of contact with $U_{p}$ is a curve of order $(2 p+2) n(n-1)$, which will be denoted by ${ }_{p} \Sigma_{u}$. It meets $\Sigma_{3}$ in $(2 p+2)(n-1)(2 n-1)$ points and ${ }_{i} L_{r}$ in $2 i(n-1)$ points. Its genus is $(4 p+4)(n-1)^{2}-6(n-1)+1$.

$\aleph_{3}$ and the $F$-chords ${ }_{i} L_{r}$ are also fundamental for the new transformation.

Corresponding to a point on ${ }_{i} L_{r}$ we get a rational curve of order $i_{n}$ lying on a rational ${ }_{n} S_{i}$ of order $2 i$, meeting $\Sigma_{3}$ in $(2 n-1) i$ points, ${ }_{j} L_{r}{ }^{\prime}$ in $\beta_{i j}$ points and ${ }_{p} \Sigma_{n}{ }^{\prime}$ in the $2 i(n-1)$ fixed points, which are the intersections of ${ }_{p} \Sigma_{n}{ }^{\prime}$ with ${ }_{r} S_{i}$.

Corresponding to ${ }_{i} L_{r}$ as a whole we get the surface ${ }_{r} S_{i}$, having $\Sigma_{3}{ }^{\prime}$ as curve of multiplicity $i$ and ${ }_{j} L_{r}{ }^{\prime}$ as line of multiplicity $\beta_{i j}$. 
Corresponding to a point on $\Sigma_{3}$ there is in general a rational curve of order $(p+1) n-2$ lying as a rational $S_{2 p}$ meeting $\Sigma_{3}{ }^{\prime}$ in $(2 p+2) n-(p+4)$ points, ${ }_{i} L_{r}^{\prime}$ in $i$ points, and ${ }_{p} \Sigma_{n}{ }^{\prime}$ in $(2 p+2)(n-1)$ points, which are the intersections of ${ }_{p} \Sigma_{n}{ }^{\prime}$ with $S_{2 p}{ }^{\prime}$.

Corresponding to $\Sigma_{3}$ as a whole we get a surface of order

$$
(4 p+4) n-(2 p+6)
$$

having $\Sigma_{3}^{\prime}$ as curve of multiplicity $(2 p+2) n-(p+4),{ }_{i} L_{r}^{\prime}$ as line of multiplicity $i(2 n-1)$ and ${ }_{p} \Sigma_{n}{ }^{\prime}$ as curve of multiplicity 2 . It will be denoted by ${ }_{p} V_{n}^{\prime}$; since it is the locus of the rational curves corresponding to the various points on $\Sigma_{3}$ it is a rational surface.

It contains, in addition, $(2 p+2)(n-1)(2 n-1)$ lines corresponding to the $(2 p+2)(n-1)(2 n-1)$ points of intersection of ${ }_{p} \Sigma_{n}$ with $\Sigma_{3}$. For each of these points on $\Sigma_{3}$ the corresponding rational curve of order $(p+1) n-2$ is degenerate and composed of a chord and a rational curve of order $(p+1) n-1$.

$\$ 9$. The full statement of the transformation is now as follows :-

Corresponding to a line in (1) we get a rational curve of order $(2 p+2) n-3$ meeting $\Sigma_{3}{ }^{\prime}$ in $(2 p+2) n-(2 p+6)$ points, ${ }_{i} L_{r}^{\prime}$ in $2 i$ points, and ${ }_{p} \Sigma_{n}^{\prime}$ in $(4 p+4)(n-1)$ points.

Curresponding to a plane in (1) we get a rational surface in (2) of order $(2 p+2) n-3$, having $\Sigma_{3}^{\prime}$ as curve of multiplicity $(p+1) n-2,{ }_{p} \Sigma_{n}{ }^{\prime}$ as simple curve, and ${ }_{i} L_{r}^{\prime}$ as line of multiplicity $i_{n}$. Such a surface possesses $(2 p+2) n(n-1)+3$ lines.

$\S 10$. Suppose spaces (1) and (2) to be superposed, the $F$-systems being distinct from one another. Certain points will coincide with their correspondents. These are the so-called double points.

The locus of the intersection of corresponding chords of $\Sigma_{3}$ and $\Sigma_{3}^{\prime}$ is a curve of order $4 p+2$, which intersects $\Sigma_{3}, \Sigma_{3}^{\prime}$ in $6 p+2$ points each and ${ }_{i} L_{r},{ }_{i} L_{r}^{\prime}$ in $2 i$ points each. If this curve meet ${ }_{p} U_{n}$ in a point $Q$, this point must also lie on ${ }_{p} U_{n}^{\prime}$, i.e. on the curve of intersection of these surfaces. The double points are therefore the points on which the curve meets the intersection of ${ }_{p} U_{n},{ }_{p} U_{n}{ }^{\prime}$.

The number is

$$
\begin{gathered}
(4 p+2)[(2 p+2) n-(2 p+4)]-(6 p+2)[(p+1) n-(p+3)] \\
-2 \Sigma \alpha_{i} i^{2}(n-1)
\end{gathered}
$$

giving $(4 p+4) n-4$. This is in accordance with the general principle that if $\lambda$ be the order of a birational reversible space transformation the number of double points is $2 \lambda+2$. 


\section{Partially Involutive Transformations.}

Suppose $T_{2 p-1}^{r}$ to be completely involutive with coincident $F$-systems. The number of double chords is $p+2$, and on each of these there lie two of the double points of $T_{s p-1}$. If the surfaces ${ }_{p} U_{n},{ }_{p} U_{n}^{\prime}$ be distinct there are an infinite number of involutive point pairs whose locus is a surface. The order of the surface is $(4 p+4) n-(4 p+6)$, and it has $\Sigma_{3}$ (the common cubic) as curve of multiplicity $(2 p+2) n-(2 p+4)$, and ${ }_{i} L_{r}$ as line of multiplicity $2 i(n-1)$.

Each of the double chords must meet the curve of intersection of ${ }_{p} U_{n},{ }_{p} U_{n}^{\prime}$ in two points, and these are double pairs both of $T_{2 p-1}$ and of the new transformation. They lie on the surface of involutive point pairs. If the transformation of the chord system of $\Sigma_{3}$ into itself possess a surface of self-corresponding chords, there will be an involutive point pair on each of its generators, namely, the double pairs of the involution determined by the two point pairs in which the chord meets ${ }_{p} U_{n},{ }_{\nu} U_{n}^{\prime}$.

\section{Completely Involutive Transformation.}

If the surfaces ${ }_{p} U_{n},{ }_{p} U_{n}^{\prime}$ also coincide, the transformation becomes involutive throughout, and the common surface ${ }_{p} U_{n}$ is a surface of double points.

\section{§11. Particular Cases.}

For the two cases in which $\breve{\searrow}_{3}, \breve{\unlhd}_{3}^{\prime}$ are degeverate $(\$ \overline{5})$ the above results are modified as follows:-

(a) $\Sigma_{3}, \Sigma_{3}^{\prime}$ composed of a line $\sigma_{1}, \sigma_{1}^{\prime}$ and a conic $\Sigma_{2}, \Sigma_{3}^{\prime}$.

The transformation is of order $(2 p+1) n-2 .{ }_{p} U_{n},{ }_{p} U_{n}^{\prime}$ are each of order $(2 p+1) n-(2 p+2)$, and have $\Sigma_{2}, \nu_{2}^{\prime}$ as curves of multiplicity $p n-1(p+1)$, and $\sigma_{1}, \sigma_{1}^{\prime}$ lines of multiplicity $n(p+1)-(p+3)$.

The $F$-curves are of order $(2 p+1) n(n-1)$, and the $F$-surfaces corresponding to them of order $(4 p+2)(n-1)$. These surfaces have $\searrow_{2}, \Sigma_{2}^{\prime}$ as curves of multiplicity $2 p(n-1)$ and $\sigma_{1}, \sigma_{1}^{\prime}$ as lines of multiplicity $(2 p+2)(n-1)$. 
The $F$-surface corresponding to $\frac{\Sigma_{2}}{\Sigma_{2}^{\prime}}$ is of order $(2 p+1) n-3$, having $\Sigma_{2}\left(\Sigma_{2}\right)$ as curves of multiplicity $p n-2$, and $\sigma_{1}^{\prime}, \sigma_{1}$ as lines of multiplicity $(p+1) n-2$.

The $F$-surfaces corresponding to $\sigma_{1}, \sigma_{1}{ }^{\prime}$ are of $\operatorname{order}(2 p+1)(n-1)$, and have $\frac{\Sigma_{2}}{\Sigma_{2}^{\prime}}$ as curves of multiplicity $n(p+1)-(p+1)$, and $\sigma_{1}, \sigma_{1}{ }^{\prime}$ as lines of multiplicity $p n-(p+1)$.

(b) $\Sigma_{3}, \Sigma_{3}^{\prime}$ composed of lines $\sigma_{1} \sigma_{2} \sigma_{3}, \sigma_{1}{ }^{\prime} \sigma_{2}{ }^{\prime} \sigma_{3}{ }^{\prime}$.

The transformation is of order $2 n p-1 .{ }_{p} U_{n},{ }_{p} U_{n}^{\prime}$ are of order $2 p n-2 p$, and have $\left(\sigma_{1} \sigma_{2} \sigma_{3}, \sigma_{1}{ }^{\prime} \sigma_{2}{ }^{\prime} \sigma_{3}{ }^{\prime}\right)$ as lines of multiplicity $p n-(p+1)$.

The $F$-curves are of order $2 p n(n-1)$, and the corresponding $F$-surfaces of order $4 p(n-1)$. These surfaces have $\sigma_{1} \sigma_{2} \sigma_{3}\left(\sigma_{1}{ }^{\prime} \sigma_{2}{ }^{\prime} \sigma_{3}{ }^{\prime}\right)$ as lines of multiplicity $2 p(n-1)$.

The $F$-surfaces corresponding to $\sigma_{1}\left(\sigma_{1}^{\prime}\right)$ are of order $2 p-2$, and the surfaces corresponding to $\sigma_{2} \sigma_{33}\left(\sigma_{2}^{\prime} \sigma_{3}^{\prime}\right)$ are of order $2 n p-2 p$. On the latter surfaces $\sigma_{1}\left(\sigma_{1}^{\prime}\right)$ are lines of multiplicity $p-1$ and $\sigma_{2}\left(\sigma_{2}\right)$ lines of multiplicity $2 n p-2 p-1$.

\section{§12. Non-Reversible Transformations.}

In the above doubly infinite series of reversible transformations the surfaces ${ }_{p} U_{n},{ }_{p} U_{n}^{\prime}$ have been similar surfaces.

We may, however, suppose them to be degenerate.

As a single example we shall take a form for ${ }_{p} U_{n}$ consisting of a ruled $S_{2 m}$ having $\Sigma_{3}$ as curve of multiplicity $m$, and a surface ${ }_{p} X_{n m}$ of order $(2 p+2) n-(2 p+4+2 m)$ having $\searrow_{3}$ as curve of multiplicity $(p+1) n-(p+3+m)$, and ${ }_{i} L_{r}$ as line of multiplicity $i(n-2)$. We shall also suppose that ${ }_{i} L_{r}$ is a line of multiplicity $i$ on $S_{y_{m}}$.

$S_{2 m}$ plays no part proper in the transformation.

${ }_{p} X_{n m}$ transforms by $T_{2 p-1}$ into a surface of order

$$
(2 p+2) n-2 p(m+1)+2 p^{2}-6,
$$

having $\Sigma_{3}^{\prime}$ as curve of multiplicity $(p+1) n-p(m+1)+p^{2}-4$, and ${ }_{i} L_{r}$ as line of multiplicity $i(n+p-m-1)$.

The order of the transformation from $(1) \rightarrow(2)$ is

$$
(2 p+2) n-(2 m+3)
$$

and from $(2) \rightarrow(1)$ it is

$$
(2 p+2) n-2 p(m-p)-5 .
$$


The $F$-curve in (1) is of order

$$
2 n^{2}(p+1)-2 n[(p+1)(p+m)+2]-(3 p+m)(p+m)+3,
$$

and the $F$-surfaces of order $4(p+1) n-4(p+m+1)$. It has $\Sigma_{3}$ as curve of multiplicity $2(p+1) n-2(p+m+1)$, and ${ }_{i} L_{r}$ as line of multiplicity $2 i(n-2)$.

The $F$-curve in (2) is of order

$$
\begin{gathered}
2 n^{2}(p+1)+p m+p+2 m-7 n+m^{2}-3 n p+4 n p^{2} \\
-2 m p^{2}+2 n m p^{2}-2 n m-7,
\end{gathered}
$$

and the $F$-surfaces of order $4(p+1) n-4(m+1) p+4 p^{2}-8$. It has $\Sigma_{3}^{\prime}$ as curve of multiplicity $2(p+1) n-2(m+1) p+2 p^{2}-4$, and ${ }_{i} L_{r}{ }^{\prime}$ as line of multiplicity $2 i(n+p-m-1)$.

The order of the $F$-surface corresponding to $\Sigma_{j}^{\prime}$ is

$$
4(p+1) n-4 m p+4 p^{2}-2 p-10,
$$

and the order of the surface which corresponds to $\Sigma_{3}$ is

$$
4(p+1) n-(4 m+2 p+6) \text {. }
$$

From this example it is evident that a complete account of the general non-reversible transformation would be of very great complexity.

Two results can, however, be stated in a simple form.

When the spaces are superposed :-

(a) The number of double pairs is in general $\lambda+\mu+2$ where $\lambda$ and $\mu$ are the orders of the transformation $(1) \rightarrow(2)$ and $(2) \rightarrow(1)$ respectively.

(b) In the partially involutive transformations the order of the surface of involutive point pairs is $\lambda+\mu-4 p$.

The corresponding results for the reversible transformation are obtained at once by putting $\lambda=\mu=(2 p+2) n-3$.

It need hardly be mentioned that the non-reversible transformations are much more numerous than the reversible transformations.

\$13. Some General Observations on the Reversible Transformations.

By assigning the value 1 to $n$ in the expression $(2 p+2) n-3$ we get $2 p-1$, and hence the base transformations are themselves the simplest of the general series of reversible transformations. 
If we assign the values 1,2 for $p$ and $n$ respectively we get the simplest of the more general series, namely, a quintic transformation. This transformation can be treated with complete generality by elementary methods. The collinear relationship between the chord systems of $\Sigma_{3}$ and $\Sigma_{3}{ }^{\prime}$ can be established directly by means of three pencils of conicoids and the point-to-point correspondence between the spaces (1) and (2) can be completed by means of a correlative transformation between the points and planes of (1) and (2).

The series given by $4 n-3(p=1)$ in general exhibits a strong analogy to another series of transformations given by Noether and de Paolis. In this latter series we have in each space a $F$-point of order $n-1$ and a $F$-curve of order $n(n-1)$ which passes through it. The bundles of lines through the $F$-points are connected by a collinear relationship. Corresponding to the $F$-points we have $F$-surfaces of order $n-1$, and to the $F$-curves conical surfaces of order $2(n-1)$ having their vertices at the $F$-points.

In the above series the cubics $\Sigma_{3}, \Sigma_{3}^{\prime}$ play rôles analogous to the $F$-points and the bundles of lines through these points to the chord systems of $\Sigma_{3}$ and $\Sigma_{3}^{\prime}$. 